\title{
Die Biopsie von Knochentumoren
}

\author{
Marcel-Philipp Henrichs, Arne Streitbürger, Ralf Dieckmann, Markus Nottrott, \\ Georg Gosheger, Jendrik Hardes
}

\section{Zusammenfassung}

Die Biopsie von Knochentumoren ist ein oftmals chirurgisch komplexer Eingriff, der den Endpunkt eines standardisierten Vorgehens zur Diagnosefindung darstellt. Indikation, OP-Planung, technisch und onkologisch saubere Durchführung sowie interdisziplinäre Diagnosefindung entscheiden darüber, ob eine korrekte Versorgung von Patienten mit Knochentumoren gelingen kann. Die Biopsie von Knochentumoren sollte daher nur von tumororthopädisch erfahrenen Operateuren vorgenommen werden.

\section{The Biopsy of Bone Tumours}

The biopsy of bone tumours is often a very complex surgical procedure and the endpoint of a standardised diagnostic algorithm. Indication and surgical planning as well as a technically and oncologically correct accomplishment of the biopsy in combination with an interdisciplinary diagnostic conference are crucial for the adequate treatment of bone tumours. Therefore biopsies should be performed by experienced surgeons with sufficient knowledge of both tumours and orthopaedics.
Biopsie bedeutet Entnahme von Gewebe aus einem lebenden Organismus zur histologischen Aufarbeitung im Rahmen der Diagnosestellung.

Meist wird ein Patient von einem niedergelassenen Kollegen zur Abklärung eines unklaren Knochenbefunds in der Klinik vorgestellt. Dieser Befund ist entweder durch Schmerzen oder Schwellung symptomatisch geworden oder im Rahmen anderweitiger Diagnostik als $\mathrm{Zu}$ fallsbefund entdeckt worden. Um nun entscheiden zu können, ob bei der vorliegenden Raumforderung eine Biopsie durchgeführt werden muss, müssen viele Informationen zusammengetragen werden. Der wichtigste Punkt hierbei ist, sich die möglichen Differenzialdiagnosen herauszuarbeiten.

Hierzu benötigt man:

1. Informationen aus Anamnese und Klinik.

2. Eine sinnvolle und ausreichende Bildgebung.

OP-JOURNAL 2010; 26: 172-176

(c) Georg Thieme Verlag KG Stuttgart · New York DOI http://dx.doi.org/10.1055/s-0030-1250539
3. Kenntnis der verschiedenen Knochentumoren mit dem Wissen, in welchem Alter welcher Tumor wahrscheinlich ist und in welchem Teil des Knochens der Tumor anzutreffen ist.

4. In manchen Fällen sind zudem Laborparameter eine sinnvolle Ergänzung.

Auf die verschiedenen Arten von primären und sekundären Knochentumoren wird an dieser Stelle nicht eingegangen, hierzu wird auf entsprechende Literatur verwiesen.

Beim Vorliegen einer Skelettläsion müssen neben Tumoren primär andere Prozesse ausgeschlossen werden. Hierzu gehören z.B. Knocheninfarkte, infektiöse Knochenerkrankungen, die Myositis ossificans und metabolische Erkrankungen des Knochens (z. B. Morbus Paget).

Sowohl die Anamnese als auch die klinische Untersuchung geben einen Hinweis darauf, ob es sich beim vorliegenden Tumor um einen bösartigen Prozess handeln könnte. Bei tastbaren Tumoren erlangt man auf diese Weise z.B. Informationen über die Wachstumsgeschwindigkeit und die Verschieblichkeit und Konsistenz der Raumforderung. Weiter- hin ist z.B. der Nachtschmerz eher typisch für entzündliche Prozesse oder für das Osteoidosteom, während ein Belastungsschmerz häufig bei osteolytischen Prozessen vorzufinden ist. Zusätzlich zur Anamnese und klinischen Untersuchung ergeben sich weitere Informationen aus dem Alter des Patienten. In Zusammenschau mit der Lage des Tumors im Knochen können so mögliche Differenzialdiagnosen weiter eingegrenzt werden.

Zur Beurteilung von Knochentumoren ist ein Röntgenbild in 2 Ebenen zwingend erforderlich.

Dieses gibt Aufschluss über die Lage des Tumors im Knochen und eine etwaige extraossäre Komponente. Als Weiteres kann beurteilt werden, ob es sich um einen osteolytischen, einen osteoblastischen oder einen gemischten Prozess handelt. Zudem ist eine Aussage über die Matrix des Tumors möglich. Oftmals kann aus diesen Informationen bereits eine Verdachtsdiagnose gestellt werden [5]. Eine weitere sehr wichtige Information sind Anzeichen für die Aggressivität des Knochentumors. Während ein Sklerosesaum um den Tumor für einen langsam wachsenden Prozess spricht, sprechen permeatives Wachstum und Zerstörung der Kortikalis sowie Fehlen eines Sklerosesaums für einen aggressiven und schnell wachsenden Tumor. Aus diesen Wachstumsmustern wurde von Lodwick eine Klassifikation [7] entwickelt (Abb. 1) [6].

\section{Grad I: Geografische Destruktion}

Langsames Tumorwachstum, fast ausschließlich bei gutartigen Tumoren, kann jedoch auch bei langsam wachsenden bösartigen Tumoren vorkommen, z.B. Chondrosarkom.

IA: Begrenzung regulär, lobuliert, scharf, keine vollständige Kompaktapenetration, immer Sklerosesaum, Kompaktaausbeu- 


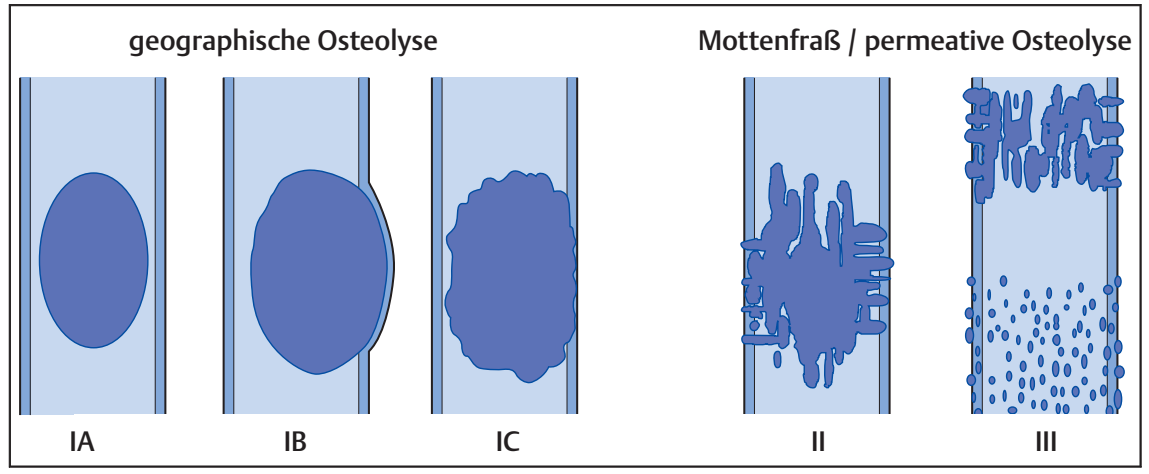

Abb. 1 Lodwick-Klassifikation (1980) (Uhl M, Herget GW. Radiologische Diagnostik von Knochentumoren. 1. Aufl. Stuttgart: Thieme; 2008 [6]).

lung $<1 \mathrm{~cm}$ möglich, klinisch meist asymptomatisch.

IB: Begrenzung regulär, lobuliert, unscharf, keine vollständige Kompaktapenetration, Sklerosesaum möglich, Kompaktaausbeulung $>1 \mathrm{~cm}$ möglich, dann aber Sklerosesaum.

IC: Begrenzung regulär, lobuliert, unscharf/zerfetzt, vollständige Kompaktapenetration, Sklerosesaum und Kompaktaausbeulung möglich.

Grad II: Immer geografische, kombiniert mit mottenfraßartiger und/oder permeativer Destruktion

Bei beschleunigtem Tumorwachstum totale Kompaktapenetration, Sklerosesaum und Kompaktaausbeulung möglich, aber ungewöhnlich. Überwiegend bei bösartigen Prozessen.

\section{Grad III: Mottenfraßartige und/oder per- meative Destruktion}

Ohne geografische Komponente bei sehr schnellem Tumorwachstum, unscharfe Begrenzung, totale Kompaktapenetration, Sklerosesaum und Kompaktaausbeulung möglich, aber ungewöhnlich. Überwiegend bei bösartigen Prozessen.

Während Grad-I-Läsionen häufig bei gutartigen Tumoren vorzufinden sind, sprechen Grad-II- und -III-Läsionen eher für einen abklärungsbedürftigen und eventuell bösartigen Prozess.

Es gibt jedoch auch gutartige Tumoren, die eine hohe lokale Aggressivität zeigen und die Kortikalis zerstören können. Hierzu zählen z.B. der Riesenzelltumor und die aneurysmatische Knochenzyste. Des Weiteren gibt es auch bösartige Tumoren mit einem langsamen Wachstumsmuster, wie z.B. das Chondrosar- kom. Auch eine akut verlaufende Osteomyelitis kann sich wie ein Grad II oder sogar III nach Lodwick darstellen [2].

Eine weitere wichtige Information des Röntgenbilds ist eine etwaige Reaktion der Knochenhaut. Hierzu zählen z. B. das Codman-Dreieck, Spiculae und das Zwiebelschalenphänomen. Eine solche Reaktion zeigt sich beim mittelbaren oder unmittelbaren Wachstum des Tumors durch die Kortikalis hindurch und ist ein Charakteristikum von bösartigen Tumoren. Periostale Reaktionen sind jedoch auch bei aggressiven gutartigen Tumoren zu erkennen.

Als weitere Notwendigkeit zur Biopsieplanung muss eine Schnittbildgebung durchgeführt werden. Hierzu stehen MRT und CT zur Verfügung.

Unserer Ansicht nach ist bei den meisten Fragestellungen primär eine MRT mit Kontrastmittel erforderlich. Diese gibt Hinweise zur Größe und Lagebeziehung des Tumors. So kann z. B. bei gelenknaher Lage geschaut werden, ob ein Gelenkserguss nachweisbar ist oder ob die extraossäre Weichteilkomponente des Tumors Kontakt zu Nerven oder Gefäßen hat. Die Weichteilkomponente des Tumors kann durch die MRT oft gut charakterisiert werden. Durch die genaue morphologische Abbildung ist oftmals eine genauere Eingrenzung des Tumors möglich. So ist z.B. der Nachweis von multiplen Zysten mit einer Spiegelbildung pathognomonisch für die aneurysmatische Knochenzyste.

Das Anreicherungsverhalten des Kontrastmittels gibt Aufschlüsse über Durchblutung und Aktivität des Prozesses. Zudem lassen sich so mit der MRT verschiedene Tumorareale abgrenzen. Diese Informationen sind für eine erfolgreiche repräsentative Biopsie unerläss- lich, denn Diagnose und Therapie richten sich nach dem bösartigsten Anteil des Tumors, eine Biopsie aus einem Nekroseareal wird hingegen keine Diagnose liefern können.

Bei Tumorverdacht sollte vor einer Biopsie immer eine Kompartment-MRT angefertigt werden. Nur wenn der gesamte Knochen mit angrenzenden Gelenken abgebildet ist, können eventuell vorhandene Skip-Metastasen erkannt werden.

Auch die CT liefert Hinweise über die Lagebeziehung des Tumors. Die Weichteilkomponente kann meistens jedoch nicht so exakt beurteilt werden wie mit der MRT. Bei Frage nach Ausdünnung der Kortikalis oder für den Nachweis von Matrixverkalkungen bei chondrogenen Tumoren ist jedoch die CT überlegen. Zudem kann auch hier durch Kontrastmittelgabe die Aktivität/Durchblutung der Raumforderung beurteilt werden.

Bei Verdacht auf einen aggressiven Tumor sollte zudem vor der Biopsie eine Skelettszintigrafie angefertigt werden. Diese gibt Auskunft über die Stoffwechselaktivität des Tumors und kann weitere Herde im Skelettsystem nachweisen. $\mathrm{Zu}$ beachten ist jedoch, dass auch gutartige Tumoren wie z.B. das Enchondrom in der Szintigrafie eine Anreicherung zeigen können.

Zur Ergänzung der Diagnostik kann in einigen Fällen eine Labordiagnostik hilfreich sein (z.B. Entzündungsparameter, Blutbild, ggf. Tumormarker).

Durch die nun vorliegenden Informationen sollte die Indikationsstellung zur Biopsie möglich sein. Wenn eine Biopsie notwendig ist, stehen generell 2 Methoden zur Verfügung.

1. Geschlossene Biopsie/Stanzbiopsie:

Diese Form der Biopsie bietet sich v.a. im Bereich des Achsenskeletts an. Vorteilhaft sind die geringe Invasivität, ein nur geringes Trauma und die Möglichkeit der ambulanten Durchführung. $\mathrm{Zu}$ bedenken ist jedoch, dass die Probenmenge häufig gering ist, sodass in einigen Fällen eine Re-Biopsie erforderlich sein kann. Wichtig ist bei der Stanzbiopsie, dass die Punktionsstelle markiert und sauber dokumentiert wird, der Punktionskanal muss im Falle einer Resektion mit entfernt werden.

2. Offene Biopsie:

Diese Methode stellt das Standardverfahren bei Skelettläsionen dar. Ein wesentlicher Vorteil ist hier die ge- 
wonnene Probenmenge und somit die höhere Wahrscheinlichkeit einer repräsentativen Probe. Zudem kann hier zwischen extra- und intraossärer Tumorkomponente unterschieden und getrennt biopsiert werden.

Bei beiden Biopsieformen muss der Zugangsweg beachtet werden, die Biopsiekanäle müssen bei der späteren Resektion mit entfernt werden [3-5].

Dies zeigt, dass der Operateur bereits vor der Biopsie wissen muss, über welchen Zugang der Tumor später reseziert werden muss. Die spätere Resektion darf durch die Wahl des Zugangs nicht erschwert werden. Bei einer etwaigen ReBiopsie muss zudem derselbe Zugang wie bei der primären Biopsie gewählt werden.

Eine voreilige Biopsie kann zur Unmöglichkeit einer extremitätenerhaltenden OP führen oder eine operative Sanierung komplett unmöglich machen!

Die Abb. 2 a und $\mathbf{b}$ zeigen solch grob fehlerhafte Biopsien. Im schlimmsten Fall kann die Gesamtprognose verschlechtert werden [1]! Empfehlungen zufolge sollte man nur Läsionen biopsieren, bei denen man auch in der Lage ist, die Tumorresektion durchzuführen [2]. Im Zweifel sollte vor einer Biopsie Kontakt mit einem Tumorzentrum aufgenommen werden und der Patient ggf. mit Unterlagen dort vorgestellt werden.

Die Abb. 3a-f zeigen Tumoren in häufiger Lokalisierung mit den jeweils geeigneten bioptischen Zugangswegen.

Wenn man sich zur offenen Biopsie entschließt und den geeigneten Zugang gewählt hat, gilt es Weiteres zu beachten. Vor einer Biopsie an Extremitäten sollte soweit möglich eine Blutsperre angelegt werden. Dies führt zu einer besseren Übersicht während der OP und verhindert eine starke Blutung und somit Kontamination des umgebenden Gewebes mit Tumorzellen. Der Hautschnitt sollte so klein wie möglich gehalten werden. Zum Vorgehen bis zum Tumor sollten scharfe Instrumente vermieden werden. Es ist auf penible Blutstillung zu achten. Gefäß-Nerven-Straßen dürfen während der Biopsie nicht dargestellt werden. Der Knochen kann sehr gut mit einer kleinen Fräse eröffnet werden. Je nach Fragestellung müssen ggf. extra- und intraossäre Komponenten gewonnen werden. Die MRT-Bildgebung sollte intraoperativ Be-

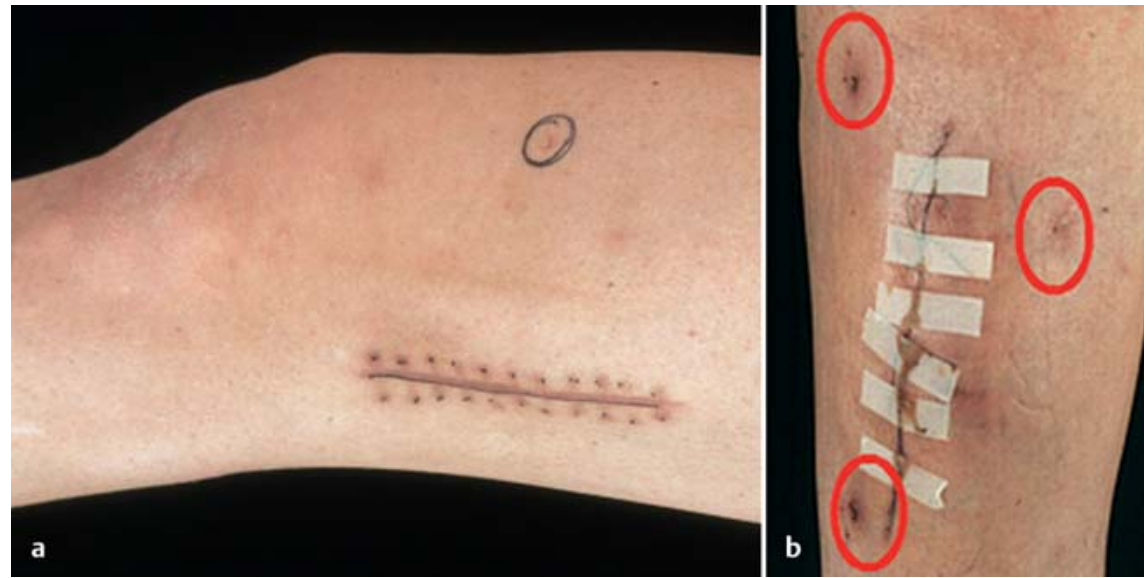

Abb. $\mathbf{2}$ a und $\mathbf{b}$ Fehlerhafte Biopsien: a Man erkennt 2 verschiedene Biopsiezugänge, die zudem sehr weit voneinander entfernt sind. Beide Zugänge müssen bei der Resektion mit entfernt werden. Der punktförmige ventrale Zugang geht direkt durch den Streckapparat, der laterale Zugang ist sehr ausgedehnt. b Lange ausgedehnte Narbe, Zugang durch den Streckapparat, die Redon-Austrittstellen sind sehr weit von der Narbe entfernt, 2 Redons sind zudem nach proximal ausgeleitet.
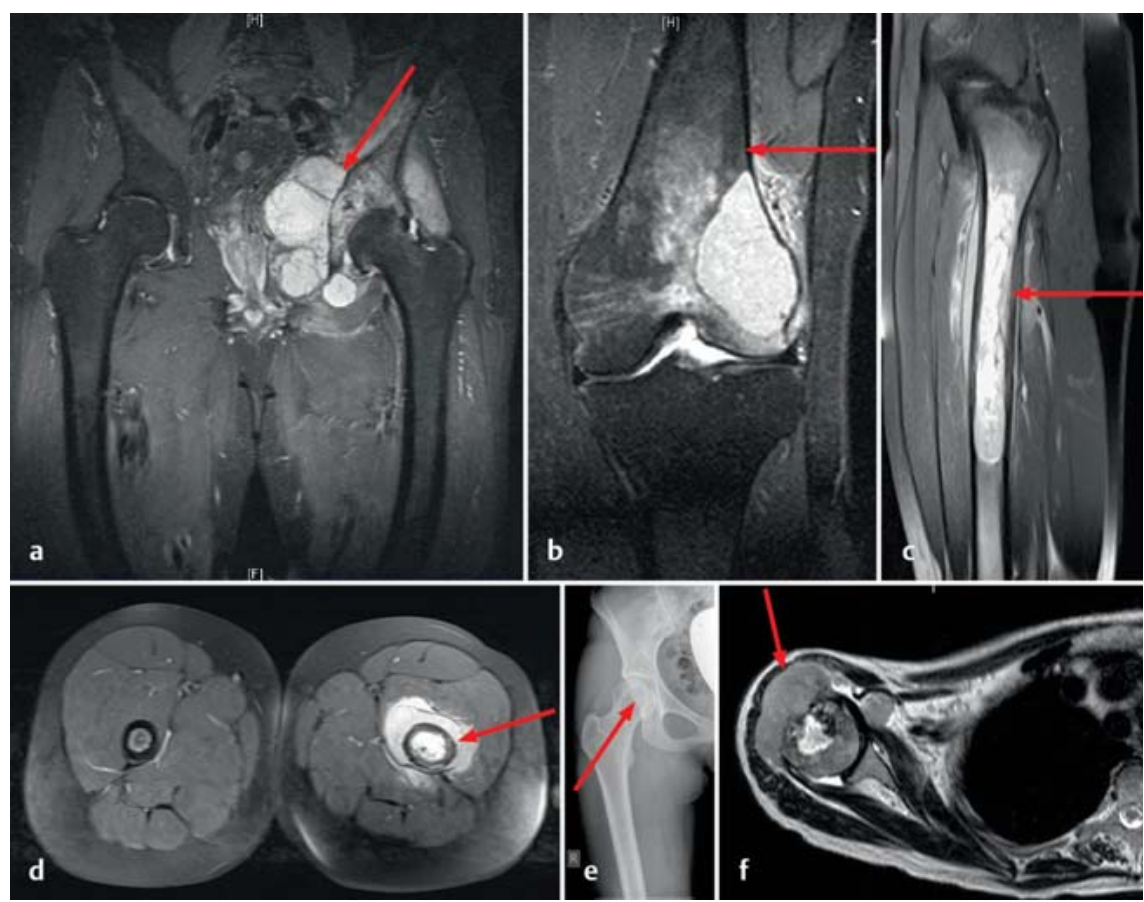

Abb. $\mathbf{3}$ a bis $\mathbf{f}$ a Chondrosarkom im Bereich des linken Hemipelvis mit großer extraossärer Komponente intra- und extrapelvin. Der Femurkopf scheint nicht beteiligt zu sein. Der Zugangsweg zur Biopsie muss im Verlauf des sog. Enneking-Zugangs liegen (Schnitt im Verlauf des Beckenkamms). Dann subperiostales Vorgehen intra- oder extrapelvin zum Tumor, die Gelenkkapsel darf nicht eröffnet werden. b Riesenzelltumor des distalen Femurs. Zugang von lateral durch den M. vastus lateralis. Der Tumor reicht nicht bis in das Gelenk. Beim Zugang ist die Ausdehnung der Gelenkrezessus zu beachten. Der Zugang muss kranial des oberen Rezessus erfolgen, das Gelenk darf unter keinen Umständen eröffnet werden! c, d Ewing-Sarkom des diaphysären Femurs. Zugang von lateral durch den M. vastus lateralis. Intra- und extraossäre Tumorkomponente. e Riesenzelltumor des proximalen Femurs. Rein intraossäre Tumormanifestation. Zugang von lateral durch den M. vastus lateralis bis auf den Knochen. Aufbohren des Knochens unterhalb des Trochanter major in Richtung Schenkelhals. Vorbohren unter Kontrolle beider Ebenen mittels Bildverstärker. Probenentnahme mittels Rangeur. Dieser Zugangsweg ermöglicht eine Probenentnahme, ohne das Gelenk eröffnen zu müssen. Der Bohrkanal sollte hierauf mit einer PalacosPlombe verschlossen werden, um eine Blutung aus dem Knochen und somit eine Verstreuung von Tumorzellen zu verhindern. f Chondrosarkom des proximalen Humerus. Zugang der Wahl ist am proximalen Humerus deltoideopektoral, transaxilläre Zugänge sind zwingend zu vermeiden, um eine Kontamination der Gefäß-Nerven-Straße zu verhindern. Glenoidale Tumoren müssen über einen anderen Zugang biopsiert werden. 


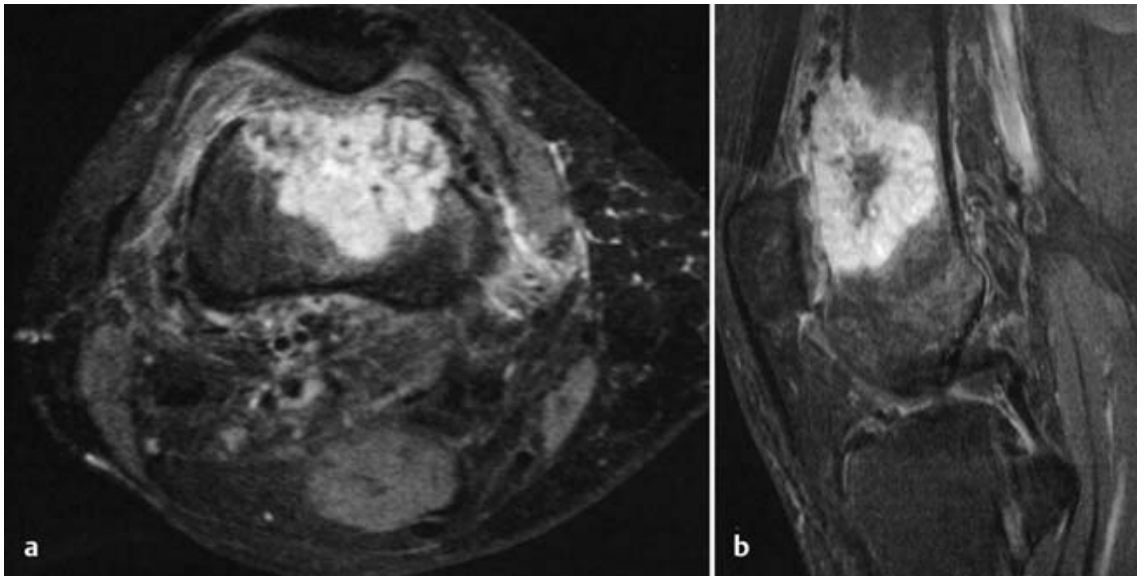

Abb. $4 \mathbf{a}$ und $\mathbf{b}$ Axiale und sagittale MRT-Schichtungen eines unklaren Tumors des distalen Femurs, gelenknahe Lage, jedoch kein Nachweis eines Gelenkergusses. Keine extraossäre Tumorkomponente. Inhomogene Kontrastmittelanreicherung. achtung finden, um eine repräsentative Probenentnahme zur gewährleisten. Das Tumorgewebe darf nicht gequetscht werden. Nach Abschluss der Probenentnahme sollte das Knochenfenster mit einer kleinen Palacosplombe verschlossen werden. Dies verhindert Blutungen aus dem Knochen und führt oft auch zu einer Stabilisierung. Nach dem Platzieren der Plombe sollte eine Drainage in die Tiefe eingelegt werden. Die Drainage sollte nach distal und direkt am Wundpol ausgeleitet werden. Faszien sollten fest vernäht werden, tief durchgreifende Nähte sind jedoch obsolet. Auch beim Rückzug sollte auf penible Blutstillung geachtet werden. Der endgültige Hautverschluss sollte mit einer fortlaufenden intrakutanen Naht vorgenommen werden. Die Extremität sollte elastokompressiv gewickelt werden, die Redon-Drainagen soll-

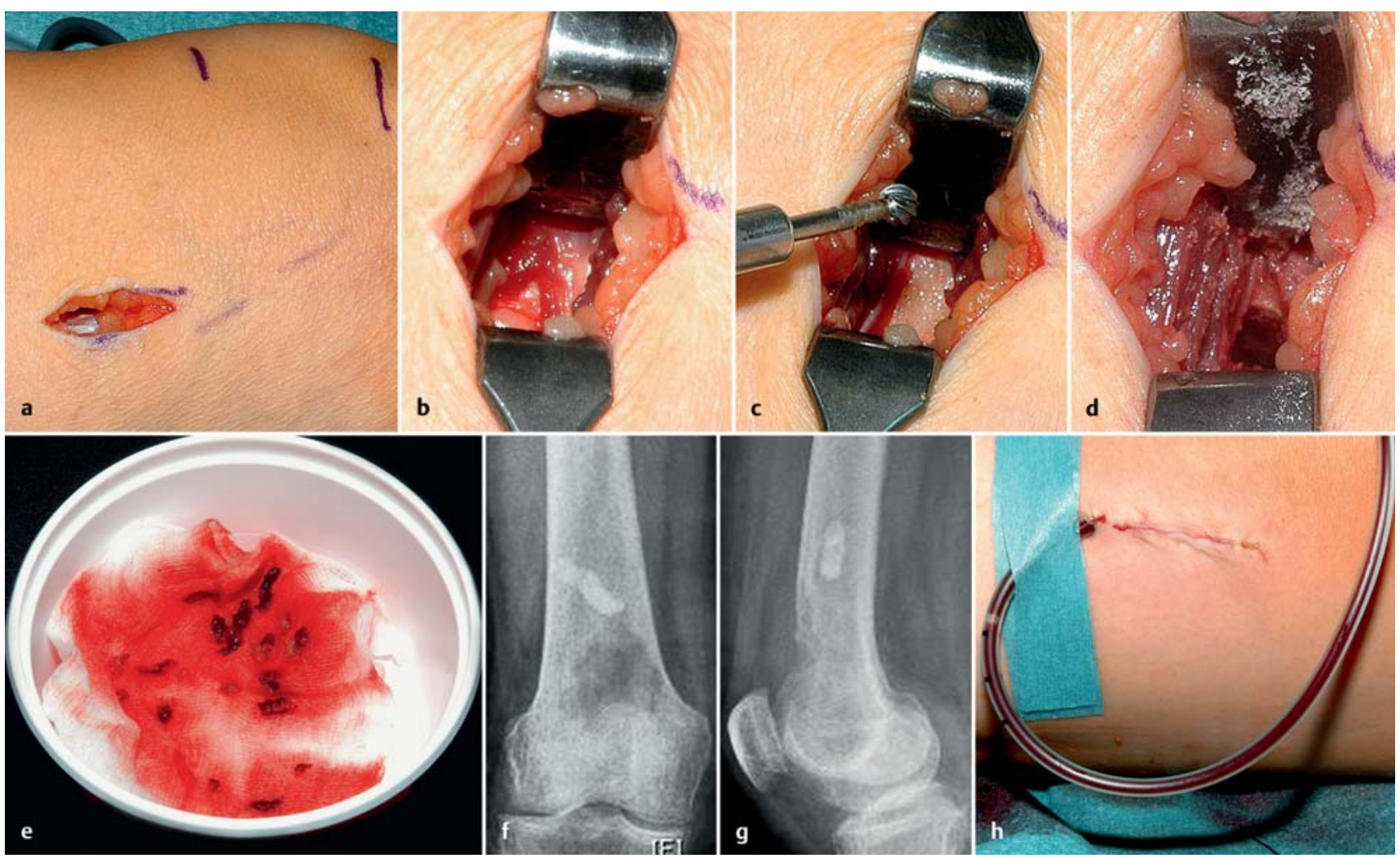

Abb. $\mathbf{5}$ a bis $\mathbf{h}$ a Vor Inzision Anzeichnen der wichtigen anatomischen Landmarken. Die hier angebrachten Markierungen zeigen den Kniegelenkspalt und die Grenze des oberen Gelenkrezessus an. Kurze Inzision von lateral durch den M. vastus lateralis. b Stumpfes Vorpräparieren mit 2 Langenbeck-Haken durch die Gewebsschichten bis Knochen-/Periostkontakt. Faszien und das Periost können unter Sicht mit einem Skalpell inzidiert werden. Ausnutzen des Hautschnitts. c Unter Darstellung des Knochens mittels 2 Langenbeck-Haken Aufbohren der Kortikalis. Dies kann mit einem Rosenbohrer erfolgen. Der Bohrer muss exakt geführt werden, um eine weitergehende Kontamination zu verhindern. Bei ausgedünnter Kortikalis kann dieser Schritt auch mit einem scharfen Löffel erfolgen. d Nach Durchbohren der Kortikalis ggf. Aufweiten des Knochenfensters. Tumorgewebe kann nun mittels Rangeur oder Löffel durch das Knochenfenster entnommen werden. e Eine repräsentative Probe beinhaltet eine ausreichende Gewebemenge aus unterschiedlichen Anteilen des Tumors. Das Gewebe sollte als Nativmaterial, also ohne Fixierung, auf einer NaCl-getränkten Kompresse umgehend mit den entsprechenden klinischen Informationen in die Pathologie geschickt werden. $\mathbf{f}, \mathbf{g}$ Röntgen a.-p. und lateral nach der Biopsie: Nach Probenentnahme sollte in den Knochen eine Palacos-Plombe eingebracht werden. Diese sollte das Knochenfenster verschließen und somit eine Blutung aus dem Knochen verhindern. h Nach Einbringen der Palacos-Plombe muss eine Redon-Drainage eingelegt werden. Diese sollte nach distal direkt am unteren Wundpol ausgeleitet werden. Danach erfolgt der schichtweise Wundverschluss. Periost und Faszien sollten gut adaptiert sein, um ein ausgedehntes Hämatom zu verhindern. Die Haut sollte mit einer Intrakutannaht verschlossen werden. 
ten auf Sog gesetzt und nicht zu früh gezogen werden. Nur so kann eine ausgeprägte Hämatombildung und somit Kontamination verhindert werden. Nach der OP ist auf körperliche Schonung des Patienten zu achten. Wenn man als Operateur den Eindruck hat, dass der Knochen durch die Biopsie und den Tumor wesentlich geschwächt ist, sollte eine Entlastung der Extremität gewährleistet werden. Im Zweifel muss bei Frakturgefahr ein Gips angelegt und ggf. Bettruhe eingehalten werden. Bei frakturgefährdeten Prozessen am Oberschenkel sollte ein Beckenbeingips angelegt und bis zur definitiven Versorgung belassen werden. Das kann im Falle eines Osteosarkoms auch mehrere Wochen bedeuten.

Die Abb. 4 und 5 zeigen eine ordnungsgemäß durchgeführte Biopsie.

Nach Durchführung der Biopsie sollte das Gewebe durch einen erfahrenen Pathologen untersucht werden.

Die Diagnosefindung sollte interdisziplinär in einer Tumorkonferenz erfolgen.

\section{Schlussfolgerung}

Wenn die Indikation zur Knochenbiopsie gegeben ist, müssen wichtige Vorgehensweisen bei der Operation beachtet werden, um die Prognose des Patienten bezüglich Funktion und Überleben nicht zu verschlechtern. Auch bei eindeutig zu wählendem Zugang müssen o.g. Arbeitsschritte Berücksichtigung finden. Bei Unklarheiten bezüglich der Biopsie ist präoperativ die Rücksprache mit einem Tumorzentrum anzuraten.

\section{Literatur}

1 Ayerza MA, Muscolo DL, Aponte-Tinao LA et al. Effect of erroneous surgical procedures on recurrence and survival rates for patients with osteosarcoma. Clin Orthop Relat Res 2006; 452: 231-235

2 Freyschmidt J, Ostertag H, Jundt G. Knochentumoren. Heidelberg: Springer; 2010

${ }^{3}$ Gosling T, Probst C, Langer F et al. [Diagnostics and treatment of primary bone tumors]. Chirurg 2010; 81: 657-678

${ }^{4}$ Leithner A, Windhager R. [Bone and soft tissue tumors: diagnostic principles]. Wien Med Wochenschr 2007; 157: 21-26

${ }^{5}$ Leithner A, Windhager $R$. [Guidelines for the biopsy of bone and soft tissue tumours]. Orthopade 2007 ; 36 : 167-174
${ }^{6}$ Uhl M, Herget GW. Radiologische Diagnostik von Knochentumoren. 1. Aufl. Stuttgart: Thieme; 2008

${ }^{7}$ Lodwick GS, Wilson AJ, Farrell C et al. Determining growth rates of focal lesions of bone from radiographs. Radiology 1980; 134: 577 583

Dr. med. Marcel-Philipp Henrichs Assistenzarzt

Dr. med. Arne Streitbürger

Oberarzt

Dr. med. Ralf Dieckmann

Assistenzarzt

Markus Nottrott, PhD

Funktionsoberarzt

Univ.-Prof. Dr. med. Georg Gosheger

Klinikdirektor

Priv.-Doz. Dr. med. Jendrik Hardes

Sektionsleiter Tumor- und

Revisionschirugie

Klinik und Poliklinik für Allgemeine Orthopädie und Tumororthopädie Universitätsklinikum Münster Albert-Schweitzer-Straße 33 48149 Münster

marcelphilipp.henrichs@ ukmuenster.de 